\title{
Fibromyalgia: psychiatrists should now be picking up the baton
}

\author{
Leigh A. Neal ${ }^{1}$
}

The Psychiatrist (2011), 35, 190-191, doi: 10.1192/pb.bp.110.030692

${ }^{1}$ Bristol Trauma Clinic

Correspondence to Leigh A. Neal (leigh.neal@btconnect.com)

First received 30 Mar 2010, final revision 11 Oct 2010, accepted 19 Nov 2010
Summary Recent functional magnetic resonance imaging (fMRI) and positron emission tomography (PET) scan studies have demonstrated the importance of the modulation of pain perception by the emotional regions of the brain in fibromyalgia. In the absence of any other objective biological abnormality, is it time to review the respective roles of primary care, psychiatry and rheumatology in the classification, diagnosis and management of fibromyalgia?

Declaration of interest None.
In clinical practice the diagnosis of fibromyalgia is often given to individuals with chronic widespread pain for which no alternative cause can be identified. Fibromyalgia syndrome, in its generally accepted manifestation, is accompanied by a combination of additional symptoms such as sleep disturbance, fatigue and anxiety, and other clinical manifestations such as depression, gastrointestinal symptoms and headache. In addition, fibromyalgia syndrome is often associated with functional somatic syndromes such as chronic fatigue syndrome and irritable bowel syndrome. ${ }^{1}$

\section{Historical context}

Traditionally, individuals with the symptoms of fibromyalgia were referred to rheumatologists because their painful, musculoskeletal symptoms were suggestive of early arthritis. When no objective evidence of organic disease could be found they would be referred to psychiatrists. From the mid-1970s some of the earliest clinical research in fibromyalgia was accomplished by close collaboration between rheumatologists and psychiatrists. Many of the key research findings at the time were published in psychiatric journals and the American Psychiatric Association was among the first medical specialty organisation to espouse fibromyalgia as a genuine condition.

In 1990, rheumatologists published diagnostic criteria for fibromyalgia, which have become generally accepted. ${ }^{2}$ These criteria include pain on both sides of the body, above and below the waist, with axial pain for at least 3 months and tenderness on manual palpation in at least 11 out of 18 defined tender points. This development became a successful stimulus to research by providing a workable definition. As a consequence, rheumatologists became the acknowledged experts in the diagnosis and management of fibromyalgia. Over the past two decades there has been an evolution in the scientific understanding of fibromyalgia, but despite intensive research efforts, no rheumatological biomarkers of fibromyalgia have been conclusively identified. Specifically, there is no agreed medical consensus for any pathophysiological abnormalities of the muscles, fibrous or connective tissues in individuals with fibromyalgia. ${ }^{3}$ As a result, an editorial in the Journal of Rheumatology has questioned whether rheumatologists should have responsibility for the management of individuals with symptoms of fibromyalgia. ${ }^{4}$

\section{Scientific basis for fibromyalgia as a psychiatric condition}

Over the past 5 years, neuroimaging studies have consistently demonstrated the involvement of centrally mediated emotional factors in regulating the enhanced perception of pain in individuals with fibromyalgia. They showed an increased activation of the emotional areas of the brain in response to non-painful pressure when compared with controls. ${ }^{5}$ The emotional areas of the brain were activated before, during and after the application of a painful stimulus. ${ }^{6}$ Central mechanisms of pain processing in the frontal cortex and the cingulate cortex were shown to play an important role in individuals with fibromyalgia. ${ }^{7}$ Symptoms of depression in this group were correlated with the magnitude of pain-evoked activation in the brain regions associated with affective pain processing. ${ }^{8}$ An 8 week, longitudinal positron emission tomography (PET) study demonstrated that, in response to an improvement in the severity of symptoms of fibromyalgia with multidisciplinary treatment, there was a concomitant increase in limbic metabolism, suggesting that the limbic system attenuates fibromyalgia symptoms. ${ }^{9}$

There is evidence from treatment and outcome studies supporting a psychiatric aetiology for fibromyalgia. A metaanalysis of 13 studies of cognitive-behavioural therapy for fibromyalgia showed that in most studies there were worthwhile improvements in pain-related behaviour, selfefficacy and overall physical function, with particular 
benefits for juveniles. ${ }^{10}$ A meta-analysis of 26 randomised controlled studies of antidepressants in fibromyalgia concluded that amitriptyline, selective serotonin reuptake inhibitors and serotonin and noradrenaline reuptake inhibitors are probably all effective in the treatment of fibromyalgia." In addition, individuals with fibromyalgia report a considerable impact on their quality of life and their perceived disability level seems to be influenced by their mental health condition. ${ }^{12}$

\section{Implications for classification, diagnosis and management}

There is probably now an adequate scientific basis and an emerging professional impetus to make it appropriate to review the present nosological status of fibromyalgia as a rheumatological condition. Pain without evidence of tissue damage, where psychological factors are judged to be important in the onset and maintenance of the pain, essentially defines a pain disorder as diagnosed by psychiatrists. The evidence from recent functional magnetic resonance imaging (fMRI) and PET scan studies raises the hypothesis that the rheumatological diagnostic criteria of fibromyalgia $^{2}$ are a subset of all pain disorders, only distinguished from other pain disorders by the circumscribed location of subjective pain with localised pressure.

The medicalisation of fibromyalgic symptoms and their management by physicians may be perpetuating a misallocation of resources and could also be impeding an individual's potential for recovery by validating a nonpsychological explanation. There is now probably sufficient evidence to re-open the debate between general practitioners, rheumatologists and psychiatrists to redefine the most appropriate care pathway for the best management of individuals with fibromyalgia.

\section{About the author}

Leigh A. Neal is a Consultant Psychiatrist, Bristol Trauma Clinic, Litfield House Medical Centre, Bristol.

\section{References:}

1 Sommer P. Fibromyalgia: a clinical update. Int Assoc Study Pain 2010; 4: $1-4$.

2 Wolfe F, Smythe HA, Yunus MB, Bennett RM, Bombardier C, Goldenberg $\mathrm{DL}$, et al. American College of Rheumatology 1990 Criteria for the classification of fibromyalgia: report of the multicenter criteria committee. Arthritis Rheum 1990; 33: 160-72.

3 Dadabhoy D, Crofford LJ, Spaeth M, Russell JI, Claw DJ. Evidence based biomarkers for fibromyalgia syndrome. Arthritis Res Ther 2008; 10: 211.

4 Shir Y, Fitzcharles M. Should rheumatologists retain ownership of fibromyalgia? J Rheumatol 2009; 36: 667-70.

5 Pujol J, Lopez-Sola M, Ortiz H, Vilanova JC, Harrison BJ, Yucel M, et al. Mapping brain response to pain in fibromyalgia patients using temporal analysis of fMRI. Plos One Epub 2009; 4: e5224.

6 Burgmer M, Pogatzki-Zahn E, Gaubitz M, Wesselock E, Heuft G, Pfleiderer B. Altered brain activity during pain processing in fibromyalgia. Neuroimage 2009; 44: 502-8.

7 Burgmer M, Pogatzki-Zahn E, Gaubitz M, Wesselock E, Heuft G, Pfleiderer B. Fibromyalgia unique temporal brain activation during experimental pain: a controlled fMRI study. J Neural Transm 2010; 117: $123-31$.

8 Giesecke T, Gracely RH, Williams DA, Geisser ME, Petzke FW. The relationship between depression, clinical pain, and experimental pain in a chronic pain cohort. Arthritis Rheum 2005; 52: 1577-84.

9 Walt B, Roebuck-Spencer T, Esposito G, Atkins F, Bleiberg J, Foster G, et al. The effects of multidisciplinary therapy on positron emission tomography of the brain in fibromyalgia: a pilot study. Rheumatol Int 2007; 11: 1019-24.

10 Bennett R, Neslon D. Cognitive behaviour therapy for fibromyalgia. Nat Clin Pract Rheumatol 2006; 2: 416-24.

11 Uceyler N, Hauser W, Sommer C. A systematic review of the effectiveness of treatment with antidepressants in fibromyalgia syndrome. Arthritis Rheum 2008; 59: 1279-98.

12 Verbunt JA, Pernot DHFM, Smeets RJEM. Disability and quality of life in patients with fibromyalgia. Health Qual Life Outcomes 2008; 6: 1-8. 